\title{
Estudio comparativo del somatotipo del rendimiento deportivo en los niños de Colombia y Ecuador
}

Comparative study of the somatotype of sports performance in boys and girls from Colombia and Ecuador

1 Carlos Alberto Sevilla Abarca https://orcid.org/0000-0002-2866-4752

Universidad Técnica de Ambato, Facultad de Ciencias Humanas y de la Educación, Maestría en Actividad Física, Tungurahua, Ecuador mayk38@hotmail.com

2 Lenin Esteban Loaiza Dávila https://orcid.org/0000-0002-5769-2795 Universidad Técnica de Ambato, Carrera de Pedagogía de la Actividad Física y Deporte, Tungurahua, Ecuador e.loaiza@uta.edu.ec

3 Paola Andrea González Castro https://orcid.org/0000-0001-5621-1809 Universidad Técnica de Ambato, Facultad de Ciencias Humanas y de la Educación, Maestría en actividad Física, Tungurahua, Ecuador paoandregonzalez13@hotmail.es

4 María Fernanda Naranjo Guevara https://orcid.org/0000-0002-1926-2612

Universidad Técnica de Ambato, Maestría en Cultura Física y Entrenamiento Deportivo, Tungurahua, Ecuador mf.naranjo@hotmail.com

Artículo de Investigación Científica y Tecnológica Enviado: 24/12/2021

Revisado: $29 / 12 / 2021$

Aceptado: $12 / 01 / 2022$

Publicado:08/03/2023

DOI: https://doi.org/10.33262/concienciadigital.v6i1.4.2029

Cítese:

Sevilla Abarca, C. A., Loaiza Dávila, L. E., González Castro, P. A., \& Naranjo Guevara, M. F. (2023). Estudio comparativo del somatotipo del rendimiento deportivo en los niños de Colombia y Ecuador. ConcienciaDigital, 6(1.4), 799-811. https://doi.org/10.33262/concienciadigital.v6i1.4.2029

CONCIENCIA DIGITAL, es una Revista Multidisciplinar, Trimestral, que se publicará en soporte electrónico tiene como misión contribuir a la formación de profesionales competentes con visión humanística y crítica que sean capaces de exponer sus resultados investigativos y científicos en la misma medida que se promueva mediante su intervención cambios positivos en la sociedad. https://concienciadigital.org

La revista es editada por la Editorial Ciencia Digital (Editorial de prestigio registrada en la Cámara Ecuatoriana de Libro con No de Afiliación 663) www.celibro.org.ec

Esta revista está protegida bajo una licencia Creative Commons Attribution Non Commercial No Derivatives 4.0 International. Copia de la licencia: http://creativecommons.org/licenses/by-nc-nd/4.0/ 
Palabras claves: somatotipo, composición corporal, rendimiento deportivo, cineantropometría
Keywords:

biotype, body composition, sport performance, cineanthropometry.
Resumen

Introducción. La gimnasia deportiva ha sido durante muchos años uno de los deportes más populares en la edad infantil, como un proceso de iniciación a la práctica deportiva, ya que la importancia de este deporte viene dada por las destrezas técnica que los niños desarrollan, la variedad de acciones motrices, combinación de movimientos y el nivel de desarrollo de capacidades físicas. Objetivo. Comparar el somatotipo de los gimnastas de iniciación de Colombia y Ecuador. Metodología. Estudio cuantitativo no experimental por alcance descriptivo comparativo y de corto transversal, aplicado en una muestra de 100 gimnastas subdividíos en 2 grupos cada uno de 50 gimnastas de Colombia y Ecuador a los cuales se recogieron medidas de peso, talla; pliegues y diámetros óseos; siguiendo el protocolo de la Sociedad Internacional para el desarrollo de la cineantropometría. Se determinó el índice de masa corporal (IMC), estado nutricional, grasa corporal total y biotipo (Endomorfía, Mesomorfia y Ectomorfia). Resultados. Los niños colombianos presentaron valores menores de IMC grasa corporal total, así como predominancia de endomorfia y mesomorfia, comparados con los niños ecuatorianos. Conclusión. Los gimnastas colombianos tienen menor grasa corporal que niños ecuatorianos, el biotipo predominante en los niños ecuatorianos es el mesomórfico, observándose un componente endomórfico menor cuanto mayor es la edad (6 a 7 años).

\section{Abstract}

Introduction. Sports gymnastics has been for many years one of the most popular sports in children, as a process of initiation to sports practice, since the importance of this sport is given by the technical skills that children develop, the variety of motor actions, combination of movements and the level of development of physical abilities. Objective. To compare the somatotype of initiation gymnasts from Colombia and Ecuador. Methodology. Non-experimental quantitative study by comparative descriptive scope and of short transversal, applied in a sample of 100 gymnasts subdivided in 2 groups each one of 50 gymnasts from Colombia and Ecuador to which weight, height; folds and bone diameters measurements were collected; 


\begin{abstract}
following the protocol of the International Society for the development of kinanthropometry. Body mass index (BMI), nutritional status, total body fat and biotype (Endomorphy, Mesomorphy and Ectomorphy) were determined. Results. Colombian children presented lower values of BMI total body fat, as well as predominance of endomorphy and mesomorphy, compared to Ecuadorian children. Conclusion. Colombian gymnasts have lower body fat than Ecuadorian children, the predominant biotype in Ecuadorian children is mesomorphic, observing a lower endomorphic component the older the age (6 to 7 years).
\end{abstract}

\title{
Introducción
}

El papel del deporte infantil y juvenil, que constituye la formación básica de la reserva deportiva de las selecciones nacionales, está aumentando actualmente (Rivas y Bailey, 2020). El alto nivel actual de rendimiento deportivo exige cada vez más a los atletas durante su entrenamiento a largo plazo basados desde un somatotipo idóneo en su formación deportiva (Cueto-Martin et al., 2017). Esto es especialmente cierto para los atletas de aquellos deportes en los que existe una especialización deportiva temprana como es el caso de la gimnasia deportiva (Vieira et al., 2021). Esta tendencia en el deporte conduce a un aumento del volumen y la intensidad de las cargas de entrenamiento y competición durante el entrenamiento de todo el año de los jóvenes atletas, un fuerte estrés psico-emocional, que puede ser la causa del desarrollo de condiciones prepatológicas y patológicas, el cese del crecimiento de los resultados deportivos Por lo tanto, un problema importante y urgente en la etapa actual de desarrollo del deporte infantil y juvenil es la preservación y el fortalecimiento de la salud de los niños y adolescentes en el proceso de las actividades deportivas (Mora-López et al., 2017).

La gimnasia deportiva ha sido durante muchos años uno de los deportes más populares en la edad infantil, como un proceso de iniciación a la práctica deportiva, ya que la importancia de este deporte viene dada por las destrezas técnica que los niños desarrollan, la variedad de acciones motrices, combinación de movimientos y el nivel de desarrollo de capacidades físicas (Shim et al., 2018).

La especificidad de este deporte es la combinación de exigencias a las condiciones físicas, con énfasis en las capacidades de flexibilidad y coordinación, y además de las grandes exigencias a los parámetros morfológicos y fisiológicos, incluido el desarrollado físico de los gimnastas. Se da preferencia a los niños y niñas del tipo de complexión delgada, 
aunque aquellos de este tipo de complexión son más propensos a un desarrollo retardado, que necesita más tiempo para formar y consolidar las habilidades motoras (AcevedoMindiola et al., 2018). Se sabe que los representantes del somatotipo asténico se caracterizan por un bajo nivel de aptitud física en comparación con otros tipos de morfología, lo que puede reducir la eficacia del entrenamiento (Díaz-Cevallos et al., 2017).

Al igual que en otros deportes, la fase de entrenamiento inicial es muy importante en el sistema de entrenamiento de los o las gimnastas. Precisamente en esta etapa es necesario llevar a cabo una mejora dirigida de las capacidades de acondicionamiento y coordinación del deportista, para sentar las bases de la técnica de manejo de aparatos y de coreografía libre, que es la condición más importante del mantenimiento del crecimiento posterior de sus resultados en la práctica competitiva a largo plazo (Estrada-Marcén et al., 2021).

Las composiciones competitivas del gimnasta están llenas de diferentes acciones técnicas con los aparatos, basados en una complejidad de los requisitos para el acondicionamiento y el entrenamiento de la coordinación en la etapa inicial del entrenamiento, que estipula la búsqueda de formas eficaces de resolver el problema de proporcionar el nivel adecuado de entrenamiento físico y de coordinación de los gimnastas, teniendo en cuenta la especificidad del desarrollo causada por las características morfológicas (Batista et al., 2019). Este tipo de deporte se caracteriza por una entrada bastante temprana a un nivel de cualificación superior, lo que lleva a una complicación constante de los elementos técnicos y su diversidad en condiciones de creciente competencia en el ámbito internacional (Caveda, 2018).

Las exigencias al nivel de aptitud física son cada vez más altas debido a la creciente complejidad de estos elementos. La complejidad de la realización del proceso de entrenamiento radica en el ámbito de la resolución de la contradicción entre la correspondencia de las variantes de piel fina de un morfotipo y la dificultad de alcanzar el nivel requerido de condicionalidad y preparación funcional de las deportistas con somatotipos similares a las exigencias estéticas de la gimnasia deportiva (Lozano-Zapata et al., 2015). En este aspecto, es prometedor identificar las peculiaridades del entrenamiento de acondicionamiento y coordinación de los gimnastas con variantes de complexión delgada para justificar posteriormente los enfoques diferenciados y, en cierta medida, individualizados, basados en la selección de la dirección y el contenido adecuados de las cargas de entrenamiento (Acevedo-Mindiola et al., 2018).

La predicción del rendimiento de los jóvenes gimnastas tiene lugar en el contexto de la formación de los procesos vitales del cuerpo, y es necesario rasgos específicos, que son poco entrenables, sin dejar de lado rasgos no determinantes que son poco susceptibles de formación. Los rasgos conservadores son relativamente fáciles de cambiar durante el proceso de mejora deportiva, ya que estos limitan el crecimiento de la deportividad y, por 
lo tanto, el proceso de selección debe basarse en estos, para el proceso de selección. La iniciación temprana en la gimnasia permite descubrir aspectos genéticamente condicionados del desarrollo físico y psicomotor (Silva et al., 2019).

Se señala que "las cualidades morfológicas y funcionales están íntimamente relacionadas y cuando se conjugan positivamente en un individuo se logra alcanzar un potencial deportivo, que se traduce en altos niveles de rendimiento" .Dentro de la cualidades morfológicas, consideramos a la longitud de las extremidades superiores y su relación existente con la estatura del individuo, que pueden ser significativas para la práctica deportiva del baloncesto, voleibol y natación, y; así como los individuos con cajas torácicas largas se inclinan hacia disciplinas deportivas de resistencia; desarrollo del sistema músculo-esquelético para deportes de potencia muscular y los individuos con longitudes de los miembros inferiores se identifican con el salto alto del gimnasta (Vernetta et al., 2017).

El biotipo de un deportista puede ser medido a través de:

1. Método fotoscópico: a través de fotografías en tres planos para determinar el somatotipo.

2. Método antropométrico: a través de pliegues, perímetros y diámetros.

3. Método antropométrico + fotoscópico: combinación de los métodos anteriores.

El método de Heath-Carter interpreta tres componentes que determinan el somatotipo del individuo, clasificándolos en endomorfos, mesomorfos o ectomorfos.

En base a la teoría planteada se establece como objetivo de investigación el comparar el somatotipo del rendimiento deportivos de niños de Colombia y Ecuador.

Las categorías biotipos o somatotípicas son la relación entre dos componentes predominantes para determinar cualitativamente las categorías somatotípicas se recurre tanto a las fórmulas matemáticas como a frases verbales o referencias que son utilizadas en forma adjunta en la determinación del biotipo a partir de fotografías o de inspección visual (Silva et al., 2019).

\section{Metodología}

La investigación responde a un enfoque cuantitativo de tipo no experimental por alcance descriptivo comparativo de corte transversal.

\section{Muestra de estudio}

Una muestra no probabilística por conveniencia que determino un total de 100 gimnastas distribuidos en 2 grupos homogéneos representantes de Colombia y Ecuador. 


\section{Técnicas e instrumentos de investigación}

Se aplicó la técnica de la observación que permitió recoger datos fórmulas para el cálculo del somatotipo de los deportistas, la misma que está basada mediante la muestra obtenida de los niños de seis años que son deportistas en gimnasia, Una vez obtenidos los valores de los componentes del biotipo se obtiene su representación gráfica a través de una somatocarta que sitúa tanto el biotipo del deportista evaluado como al parámetro del referente ideal, mediante un eje de coordenadas estableciéndose así una comparativa.

Los instrumentos que se utilizan para la evaluación del biotipo del gimnasta son:

- Un lápiz demográfico para efectuar las marcaciones corporales.

- Un calibrador de pliegues cutáneos.

- Una plataforma para medir el peso corporal.

- Un estadiómetro con precisión de fracciones de $0,1 \mathrm{~cm}$ para medir la estatura de los sujetos.

- Una cinta métrica inextensible milimetrada con precisión de 0,1 centímetro para medir las circunferencias.

- Un antropómetro para la determinación de diámetros, con precisión $\pm 1 \mathrm{~mm}$ y (g) planilla proforma de antropometría para la anotación de las medidas.

Medidas antropométricas más utilizadas en el biotipo de un deportista

A continuación, se describe los cinco tipos de medidas más utilizadas casi siempre

1. Peso: Es la medición de la masa corporal total. $\mathrm{Su}$ apreciación es relativamente fácil, pero se debe tener en cuenta que durante el día una persona adulta puede variar el peso hasta dos (2) $\mathrm{Kg}$.

Los valores más estables según Carvajal-Veitía (2017) se obtienen en ayunas posterior a la evacuación. Cada uno de los sujetos se coloca de pie en forma erecta al centro de la plataforma, descalzo, con la menor cantidad de ropa posible, evitando el contacto del cuerpo con objetos a su alrededor

1. Pliegues o panículos adiposos: Gómez-landero (2010) se considera a la medida de espesor de dos pliegues de piel y de grasa subcutánea. Los pliegues cutáneos son los siguientes: (a) Tríceps, (b), Supraespinal y (c) Subescapular.

El lugar del pliegue del tríceps está en un punto medio entre el acromion y la cabeza proximal del radio con la extremidad superior, relajado con leve rotación externa del hombro. El pliegue supraespinal, está localizado en la intersección formada por la línea del borde superior del íleon y una línea imaginaria que va desde la espina ilíaca anterosuperior derecha hasta el borde axilar anterior. Se sigue la línea del pliegue medialmente 
hacia abajo formando un ángulo alrededor de $45^{\circ}$ con la horizontal. El pliegue subescapular está ubicado en el ángulo inferior de la escápula en dirección hacia abajo y hacia fuera, formando un ángulo de $45^{\circ}$ con la horizontal.

\section{Resultados}

Este estudio presenta valores poco inferiores a los encontrados en la medición antropométrica a nivel mundial con respecto a la bibliografía con niños de edades similares y modalidades de deporte en gimnasia.

En la categoría de mujeres, los datos son ligeramente más variables, ya que en función de la modalidad deportiva, las niñas pueden ser superiores como es en el caso de la gimnasia, que a continuación se presenta las diferencias en la composición corporal y somatotipo de niñas y niños de seis años de Ecuador y Colombia, que para realizar un adecuado estudio de la composición corporal y el cálculo del biotipo o somatotipo de los niños y niñas se utilizó el método antropométrico de Heath y Carter para este estudio del cual se ha trabajado con una muestra de 50 atletas divididos en 22 niñas y 28 niños. En este estudio se ejecutó valores promedios de los indicadores como por ejemplo de los para las niñas de Colombia se tiene un valor promedio de peso de $20,9 \mathrm{~kg}$; en estatura de $112,1 \mathrm{~cm}$. Los valores se muestran en la siguiente tabla:

Tabla 1

Indicadores antropométricos

\begin{tabular}{ccccc}
\hline Sexo & F & M & F & M \\
\hline Cantidad & 22 & 28 & 22 & 28 \\
Nacionalidad & Colombiana & \multicolumn{2}{c}{ Ecuatoriana } \\
Edad (Años) & 6 & 6 & 6 & 6 \\
Peso (Kg) & 20,9 & 23,2 & 18,6 & 21,6 \\
Estatura (cm) & 112,1 & 114 & 102 & 110 \\
PI. Tríceps (mm) & 12,4 & 16,5 & 11,3 & 15,5 \\
PL. Subescapular (mm) & 6,3 & 7 & 5,8 & 6,8 \\
PI. Pantorrilla (mm) & 6,1 & 6,5 & 5,7 & 6,2 \\
PI. Supraespinal (mm) & 38,3 & 38,9 & 35,1 & 36,5 \\
P. Brazo flexionado (cm) & 8,7 & 9,9 & 7,1 & 8,9 \\
P. Pantorrilla (cm) & 61,8 & 65 & 59,4 & 60,5 \\
D. Bicondilar femur (cm) & 58 & 62 & 55 & 61 \\
D. Bicondilar humero (cm) & 6 & 6,5 & 5,3 & 6,4 \\
\hline
\end{tabular}


Tabla 2

Cálculo de la ENDOMORFIA

\begin{tabular}{ccccc}
\hline Mediciones & Ind.I & Ind II & Ind.I & Ind II \\
\hline Estatura & 112,1 & 114 & 102 & 110 \\
PI. Tríceps & 12,4 & 16,5 & 11,3 & 15,5 \\
Pl. Subescapular (mm) & 6,3 & 7 & 5,8 & 6,8 \\
PI. Supraespinal (mm) & 38,3 & 38,9 & 35,1 & 36,5 \\
Suma de Pliegues & 57 & 62,4 & 52,2 & 58,8 \\
170,18/Estatura & 1,52 & 1,49 & 1,67 & 1,55 \\
\hline ENDO $=$ & 6,321 & 6,747 & 5,920 & 6,465 \\
\hline
\end{tabular}

Nota: $E N D O=0,0000014 * X^{3}-0,00068 * X^{2}+0,1451 * X$

\section{Tabla 3}

Calculo de la MESOMORFIA

\begin{tabular}{ccccc}
\hline Mediciones & Ind.I & Ind II & Ind.I & Ind II \\
\hline Estatura (cm) & 112,1 & 114 & 102 & 110 \\
PI. Tríceps (mm) & 12,4 & 16,5 & 11,3 & 15,5 \\
PI. Pantorrilla (mm) & 6,1 & 6,5 & 5,7 & 6,2 \\
P. Brazo flexionado (cm) & 8,7 & 9,9 & 7,1 & 8,9 \\
P. Pantorrilla (cm) & 61,8 & 65 & 59,4 & 60,5 \\
D. Bicondilar femur (cm) & 58 & 62 & 55 & 61 \\
D. Bicondilar húmero (cm) & 6 & 6,5 & 5,3 & 6,4 \\
\hline MESO & 41,07497 & 44,31635 & 39,33439 & 43,26468 \\
\hline
\end{tabular}

Nota: $M E S O=0,858 * H+0,601 * F+0,161 * P-0,131 * E+4,5$

Tabla 4

Calculo de la ECTOMORFIA

\begin{tabular}{ccccc}
\hline & Ind.I & Ind II & Ind.I & Ind II \\
\hline Estatura (cm) & 112,1 & 114 & 102 & 110 \\
Peso (Kg) & 20,9 & 23,2 & 18,6 & 21,6 \\
Raíz Cubica & 2,755 & 2,852 & 2,650 & 2,785 \\
IP & 40,696 & 39,971 & 38,497 & 39,498 \\
\hline ECTO & 1,21 & 0,68 & $-0,40$ & 0,33
\end{tabular}

Nota. ECTO $=0.732 * I P-28,58$ 
Para el gráfico se utilizó las siguientes formulas

\section{Tabla 5}

\begin{tabular}{ccccc}
\multicolumn{5}{c}{ Listado de fórmulas } \\
\hline ECTO & 1,210 & 0,679 & $-0,400$ & 0,333 \\
ENDO $=$ & 6,321 & 6,747 & 5,920 & 6,465 \\
MESO & 41,075 & 44,316 & 39,334 & 43,265 \\
X $=$ & $-5,111$ & $-6,068$ & $-6,321$ & $-6,133$ \\
Y $=$ & 74,619 & 81,207 & 73,148 & 79,731 \\
\hline
\end{tabular}

Nota: $X=E C T O-E N D O$

$$
Y=2 * M E S O-(E C T O+E N D O)
$$

Los datos de los componentes del Somatotipo atendiendo las diferencias entre colombianos - ecuatorianos para la edad de 6 años se presentan en los siguientes gráficos, respectivamente.

\section{Figura 1}

Gráfico de niños-niñas de 6 años de Colombia

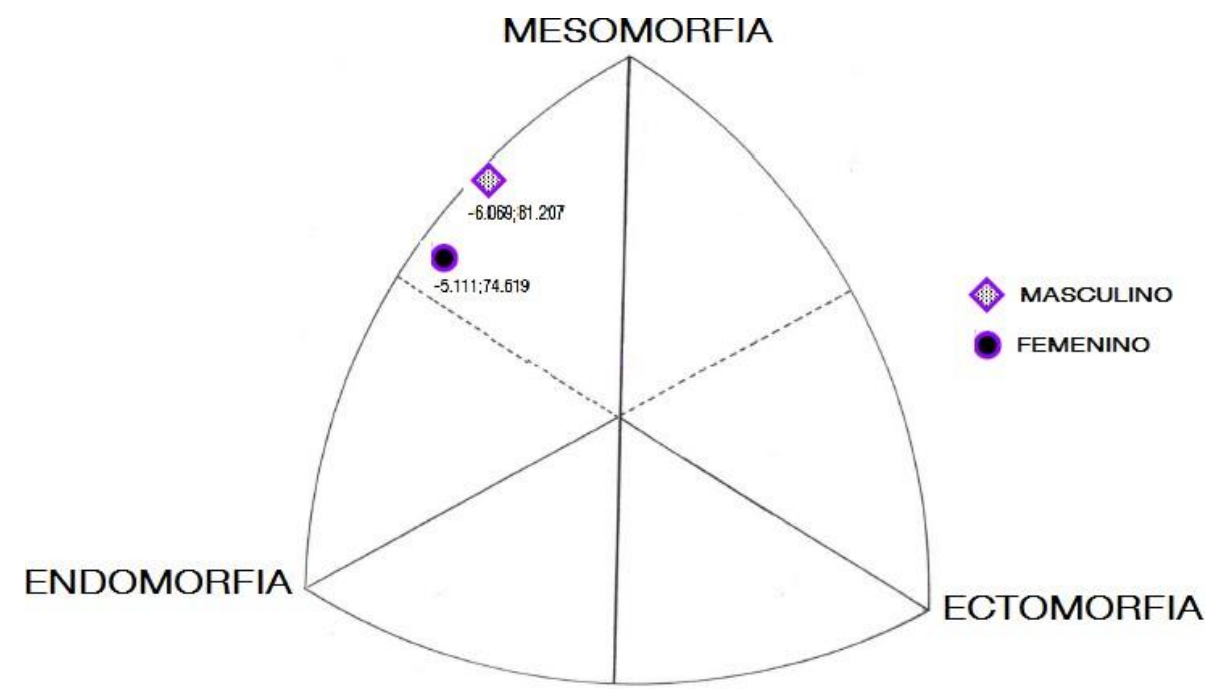




\section{Figura 2}

Niños -niñas de 6 años de Ecuador

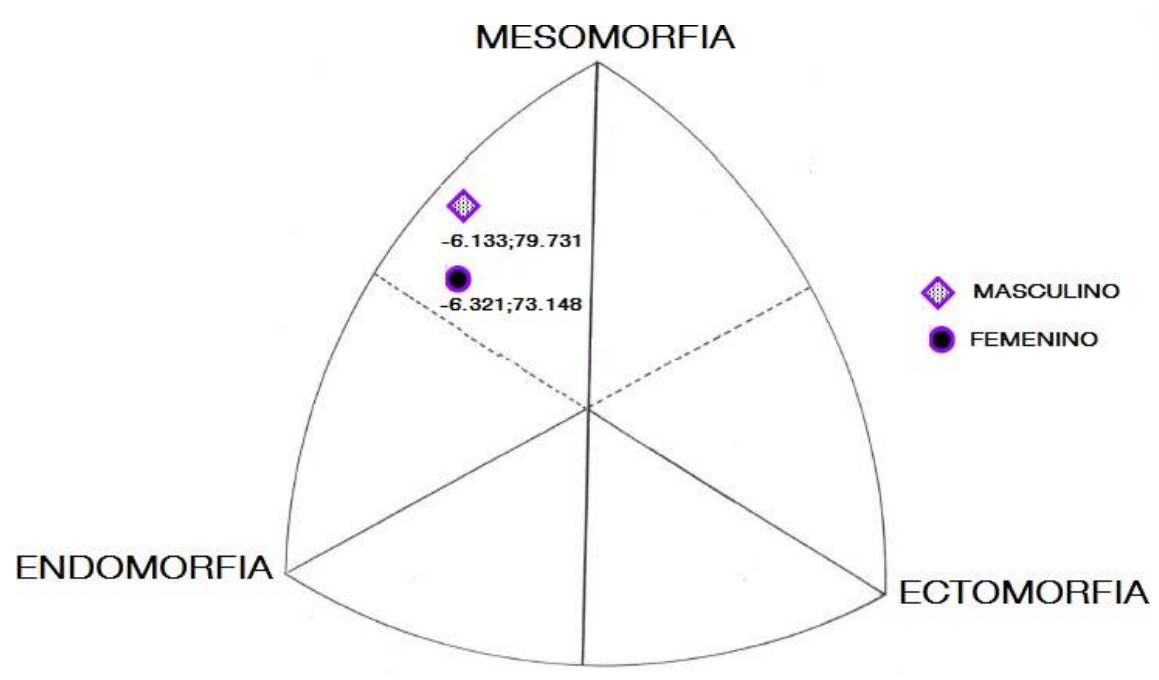

\section{Conclusiones}

- Los gimnastas ecuatorianos se caracterizan por un somatotipo mesoendomorfo, a diferencia de los gimnastas colombianos presentan como somatotipo predominante al endomesomórfico, información que el entrenador debe tomar en cuenta al inicio y durante la planificación del entrenamiento de desarrollo de capacidades física.

- En relación con el IMC y grasa corporal, la muestra de gimnastas colombianos, presento menores índices y porcentajes en relación con los gimnastas ecuatorianos, determinando un menor potencial corporal para la práctica de la gimnasia deportiva.

\section{Referencias bibliográficas}

Acevedo Mindiola, A., Bustos Viviescas, B., \& Lozano Zapata, R. (2018). Composición corporal y somatotipo de gimnastas Norte de Santander que participaron en los XX Juegos Nacionales (original). Olimpia: Publicación Científica de La Facultad de Cultura Física de La Universidad de Granma, 15(47), 117-128.

Batista, A., Garganta, R., \& Ávila-Carvalho, L. (2019). Morphological characteristics and biological maturation of Brazilian and Portuguese gymnasts. International Journal of Morphology, 37(2), 561-567. https://doi.org/10.4067/S071795022019000200561 
Carvajal Veitía, W. (2017). Contribución de la bioantropología del deporte al desarrollo del alto rendimiento y sus principales hitos en Cuba. Anales de Antropología, 51(2), 203-216. https://doi.org/10.1016/j.antro.2017.03.006

Caveda, D. L. (2018). El profesor en la iniciación deportiva y la evaluación de su desempeño El profesor en la iniciación deportiva y la evaluación de su desempeño The professor in sports initiation and the evaluation of his performance $O$ professor de iniciação esportiva e a a. February.

Cueto-Martin, B., Morales Ortiz, E., Burgueno, R., \& Medina Casaubon, J. (2017). M.A.M.I. Deporte: an Active Methodology To Improve Initiation To Sport. Preliminary Study. Journal of Sport and Health Research, 9(1), 121-128.

Díaz Cevallos, A. C., Ruiz Villacrés, H. D., \& Monroy Antón, A. J. (2017). Relación del somatotipo y las alteraciones posturales podológicas del arco plantar. Revista Cubana de Investigaciones Biomédicas, 36(2), 178-185.

Estrada-Marcén, N., Redondo-Laencina, P., Casterad-Seral, J., \& Cid-Yagüe, L. (2021). Tratamiento De La Lateralidad En Gimnasia Rítmica: Estudio Centrado En

Gómez-landero, L. A. (2010). Somatotipo y composición corporal en gimnastas de Trampolín masculino español de alto nivel Redalyc Sistema de Información Científica Gómez-Landero Rodríguez, Luís Arturo; Vernetta Santana, Mercedes; López Bedoya, Jesús Somatotipo y composición corporal en gimnastas de Trampolín masculino español de alto nivel RICYDE. Revista Internacional de Ciencias del Deporte, Vol. VI, Núm. 19, abril-sin Ramón Cantó Alcaraz España. April 2014.

Lozano Zapata, R. E., Acevedo Mindiola, A. A., \& Bustos Viviescas, B. J. (2015). Composición corporal y somatotipo de los deportistas participaron en los xx juegos nacionales 2015, Colombia. Revista Digital de Semilleros de Investigación REDSI, $1-11$.

Mora López, D., García Pinillos, F., \& Latorre Román, P. (2017). Actividad física, condición física y salud en niños preescolares. Estudio de revisión narrativa. EmásF: Revista Digital de Educación Física, 45(45), 105-123.

Rivas-Martínez, H., \& Bailey-Moreno, J. (2020). Aportaciones del fútbol en la formación infantil y juvenil en una comunidad vulnerable (Soccer contributions to child and youth training in a vulnerable community). Retos, 39, 276-283. https://doi.org/10.47197/retos.v0i39.78491

Shim, H., Shin, N., Stern, A., Aharon, S., Binyamin, T., Karmi, A., Rotem, D., Etgar, L., Porath, D., Pradhan, B., Kumar, G. S., Sain, S., Dalui, A., Ghorai, U. K., Pradhan, S. K., Acharya, S., Quan, L. N., Rand, B. P., Friend, R. H., ... Gmbh, Z. (2018). No 
主観的健康感を中心とした在宅高齢者における 健康関連指標に関する共分 散構造分析Title. Advanced Optical Materials, 10(1), 1-9.

Silva, C. C., Silva, L. F., Santos, C. R., Goldberg, T. B. L., Ramos, S. P., \& Venancio, E. J. (2019). Genetic polymorphism on the flexibility of elite rhythmic gymnasts: State of art. Apunts Medicina de l'Esport, 54(201), 27-35. https://doi.org/10.1016/j.apunts.2018.10.001

Vernetta, M., Montosa, I., Beas-Jiménez, J., \& López-Bedoya, J. (2017). Batería Funcional ARISTO en Gimnasia Rítmica: protocolo de test específicos para la evaluación de jóvenes gimnastas en un ámbito de entrenamiento saludable. Revista Andaluza de Medicina Del Deporte, 10(3), 112-119. https://doi.org/10.1016/j.ramd.2017.02.001

Vieira, F. A., Romário, W., \& Menezes, P. (2021). El discurso de tres entrenadores escolares sobre la iniciación y especialización del baloncesto The speech of three school coaches on basketball initiation and specialization. 8(3), 1171-1180.

\section{Liencia}


El artículo que se publica es de exclusiva responsabilidad de los autores y no necesariamente reflejan el pensamiento de la Revista Conciencia Digital.

\section{Ciencia \\ LDigital}

El artículo queda en propiedad de la revista y, por tanto, su publicación parcial y/o total en otro medio tiene que ser autorizado por el director de la Revista Conciencia Digital.
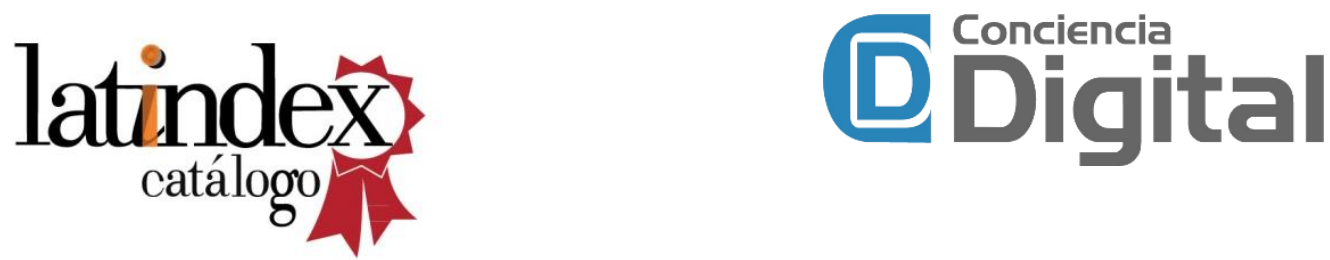

Indexaciones

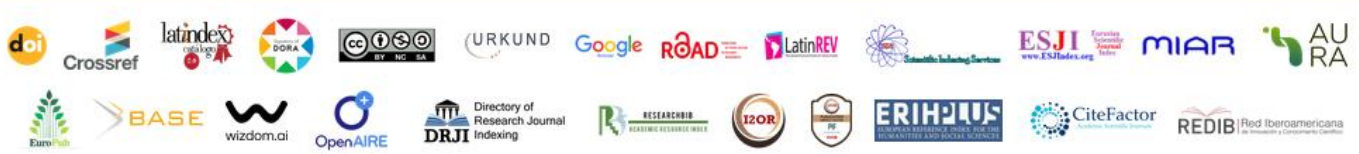

